\title{
Mental health advocacy on Twitter: positioning in Depression Awareness Week tweets
}

\author{
Nelya Koteyko \& Dimitrinka Atanasova
}

\section{Introduction}

Depressive disorders are experienced by nearly one in five UK adults (National Institute for Health and Care Excellence, 2015) and are estimated to affect over 300 million people worldwide (World Health Organization, 2017). While social support is instrumental in helping the sufferers cope, stigma concerns often discourage people from discussing the symptoms and seeking help face-to-face. Social media, therefore, has emerged as a useful platform for communication and interaction on health topics including stigmatised conditions as it reduces some of the negative connotations of face-to-face disclosure (Jamison-Powell et al, 2012). Both individuals and organisations in the health- and social care sectors actively use commercially available platforms such as Facebook (authors/reference after review) and Twitter in order to provide support, raise awareness and campaign to improve attitudes towards depression. Participants with depression specifically prefer Twitter to Facebook because of the loose social connection that allows them to tweet more openly (Park et al, 2012). Founded in 2006, Twitter represents a mode of social media known as micro-blogging - an internet-based service in which (1) users have a public profile for posting short messages or updates; (2) such messages are publicly aggregated across users, and (3) users can decide whose messages they wish to receive but not necessarily who can receive their messages, that is following each other is not necessarily mutual (Murthy, 2012). Twitter messages are short - the character limit was 140 characters until $2017^{1}$. The hashtag symbol \# allows tagging tweets with keywords that are searchable and followable (Zappavigna, 2015). Taken together, these micro-blogging features ${ }^{2}$ allow an array of communicative functions - for example, allowing individuals and organisations to use Twitter 'as a broadcast medium, marketing channel, diary, social platform, and news source' (Marwick \& boyd, 2010: 9). While media studies and sociology research has examined

\footnotetext{
1 The character limit was doubled in 2017, after our data collection had taken place.

2 Twitter also offers the facility of directed messages which are only visible to the sender and receiver, and 'mentions' (those featuring another user's @-prefixed name) which are visible to all users.
} 
the uses and functions of Twitter for organisational health communication (e.g. Park et al, 2012), little is known about how the features of this platform are shaping the discursive practices of producing and consuming patient advocacy content by individuals. From the discursive perspective on mental health that foregrounds the links between language, collective identity, and social change (Crowe, 2000; Galasinski, 2008), Twitter messages about depression represent an opportunity to study how first person accounts by individuals who experience mental distress are constructed and used in the social media context as part of social activism projects (Jones, 2015). While illness narratives (Bury, 2001) are traditionally elicited via research interviews or diaries, tweets are produced within different medium factors of computer-mediated communication (Herring, 2007) and for networked audiences (boyd and Heer, 2006; Sergeant and Tagg, 2014).

The study focuses on tweets circulated during one of the key annual advocacy events Depression Awareness Week. Depression Awareness Week was devised to highlight the help and support available for people afflicted by this mental health condition. In 2016, when this study started, the event received widespread attention across social media platforms. In the UK, 2,855 tweets were posted under the hashtag \#depression during the period of 18-22 April 2016. Blurt charity (2016) was one of the UK organisations that harnessed the power of social media during this period to reveal what living with depression is like and to reduce the stigma surrounding mental illness:

We're determined to challenge the stigma around what depression 'should' look like, and show the world that ANYONE can be affected by poor mental health. [...] We're asking people to share their insights and experiences of depression - we want to highlight the reality of depression, the impact depression has had on our lives, giving people a chance to share 'what you don't see'. We know [sic] going 'public' about your condition can feel scary, but sharing your experiences so boldly and visibly helps others and directly challenges the stigma around poor mental health. [...] Let's 'Blurt it out' and start conversations about the reality of living with depression.

Responses to this campaign provide a rich resource for examining how individuals with depression use Twitter to perform acts of public self-disclosure by providing accounts of their 
everyday experiences. Specifically, our study seeks to answer the following questions: (1) What semiotic resources are used by campaign participants to disclose depression and describe their experiences of living with the condition? (2) How do campaign participants position themselves and their audiences in this process? (3) How are these mental health advocacy strategies shaped by the affordances of the micro-blogging platform? Given that advocacy actors seek visibility it is essential to examine both linguistic and visual practices (Adami and Jewitt, 2016). On the theoretical level, we therefore emphasize the role of images as well as hashtags and hyperlinks in the production and circulation of digital illness narratives.

\section{Background: Identity disclosure in social movements}

In recent years, mental illness disorders have been increasingly medicalized in part via the media framing of mental illness as a brain disease as opposed to a reaction to or consequence of psychosocial factors (Horwitz \& Wakefield, 2007). On the one hand, medicalization or framing mental distress as a medical problem can be aimed at reducing stigma in that individuals suffering from a physical disease are not to blame for their affliction (Reali et al, 2016). On the other hand, however, such biogenetic explanation may inadvertently reinforce stereotypes suggesting that people with mental illness have no control over their behavior (Read \& Law, 1999), and increase the desire for social distance (Kvaale et al, 2013). Feelings of stigmatisation are not always a result of direct discrimination but can also occur when individuals attempt to manage information about their condition as part of social networks, which involves stressful decisions about disclosing a condition to friends, family and employers (Sokratis et al, 2004). To counteract such stereotypes and invisibility resulting from fear to disclose a stigmatized condition, patient advocacy organizations have been promoting acts of public self-disclosure.

According to Whittier (2012), the act of public self-disclosure, or 'coming out' is an instance of how identity disclosure is linked to social change outside of more conventionally defined collective action. The strategy has been adopted by individuals outside of social movements in order to make political statements of their own and influence their social networks. Whittier (2012) draws examples from the work of the feminist movements for legalization of abortion and 
against rape who developed the 'speak out' vocabulary to reveal everyday experiences of ordinary women and challenge their invisibility. In such instances, speaking about personal circumstances 'acquired political meaning both because of its effects on individual emotion (reducing shame, promoting pride), and because the individual was aligned with a collectivity' (Whittier, 2012: 149). By disclosing individual experiences and identities, even people who did not directly participate in the social movement were able to declare their allegiance to it and in this way challenge dominant representations of their group within their circles.

Whereas the tactics of coming out by feminist movements were carried out largely through public events and public cultural projects, in the recent years the visibility politics described by Whittier (2012) have been increasingly moving online. Tembeck (2016) examines how social media bloggers create and circulate selfies of ill health, using the language of activism such as 'empowerment', 'starting a movement' and 'countering stigma'. She takes as a case study Karolyn Gehrig's \#HospitalGlam selfies and shows how posting such selfies on social media enables individuals to 'come out' as invisibly ill. Jones (2015) highlights the potential of online storytelling for social activism through the analysis of 'It Gets Better' videos - a successful campaign for 'at risk' LGBT adolescents that led to the creation and circulation of over 50,000 YouTube videos. Driven by temporary online collaborations among individuals and 'personalised engagement' with protest waves (e.g. the \#metoo movement) such digitally mediated activist practices are best described within the framework of 'connective action' (Bennet \& Segerberg, 2013), which complements rather than replaces collective action (Meikle, 2018).

\section{Conceptual framework: Illness narratives as networked stories}

Research on the forms and functions of illness narratives expanded rapidly during the last decades of the twentieth century (Bury, 2001; Charmaz, 2002), driven in part by the need to recognise lay experiences and individual suffering in everyday contexts. Social media campaigns that invite people to tell stories of their personal experiences via micro-blogging platforms capitalise on the potential of stories to bring the listeners into the private world of the storyteller and reveal subjective experiences. Provision of detailed, mundane and personal content via 
online (micro-)blogging creates authenticity, a feature of autobiographical narratives that can be traced back to traditional, offline diaries. Such diaries have been studied as important sociological material for many years since the development of the pioneering approach to 'personal documents' by the Chicago School sociologists (Thomas \& Znaniecki, 1958). However, told and circulated via social media, 'networked narratives' (Page et al, 2013) have structural and situational differences from the illness narratives produced via the mode of diary writing or elicited through face-to-face interviews (Bury, 2001).

Firstly, the increasing use of social media for the purpose of patient advocacy and health promotion needs to be understood in the context of digital affordances of the sites, identified by boyd (2010) as persistence, replicability, scalability, and searchability. Taken together these affordances underscore the fact that the shared online content is routinely saved and archived, replicated, as well as searched and retrieved by the individuals who join the social networking platforms. Scalability, for example, defined by boyd (2010) as the potential visibility of content is deemed to have implications for public health promotion (Neuhauser \& Kreps, 2003) as it transforms what would have been 'private' health entries in a food or exercise journal into interactive 'public' disclosures. In the case of Twitter, the content of posts can be read by both the poster's immediate addressee (addressed directly via @), by the user's follower network and anyone using the same hashtag, and 'overheard' by the public at large (drawing on Goffman's (1981) distinction between ratified and unratified audiences). As a result, as Marwick and boyd (2010: 99) note, 'it is virtually impossible for Twitter users to account for their potential audience, let alone actual readers'. Sociolinguistic research therefore has examined how users of social media sites create their posts for an 'imagined audience' (Marwick \& boyd, 2010; Seargeant \& Tagg, 2014) focusing on strategies of self-presentation and impression management (Bolander \& Locher, 2010) as well as the issues of authenticity (Marwick \& boyd, 2010).

Secondly, online narratives (such as those found in tweets, Facebook and forum posts) do not conform to the structuralist narrative paradigm (e.g. Labov, 1972), which restricts the definition of narrative to long, teller-led, single-teller accounts of distant, past events or of one's 
autobiographical story (Georgakopoulou, 2015). What emerged as a 'small stories' approach, recognises 'a-typical', 'non-canonical' tellings in group settings (Georgakopoulou, 2015: 2), which are normally small in length, occur within everyday life situations and deal with recent, ongoing or still unfolding events (e.g. 'breaking news' stories, see also Page, 2010, 2012). Another type of small story highly relevant to the social media setting is a 'shared story' - account of shared or known events that are collaboratively told and retold and range from 'full-fledged tellings to more or less elliplical and skeletal tellings' (De Fina \& Georgakopoulou, 2012: 109). Drawing on the contextualised view of narration developed by Ochs and Capp (2001), this approach emphasises the role of narratives in the construction of different identities as speakers position themselves and others through language use. The act of positioning implies ascribing or denying certain rights, duties, and relationships that are normatively associated with that position (Harré, 2012). It is a dynamic process in which the speakers 'actively and agentively select, resist and revisit positions' (Georgakopoulou, 2007: 123) both in relation to the interactional moment and 'in relation to the dominant discourses which constitute their context (Bamberg \& Georgakopoulou, 2008: 379). For example, research on illness narratives has shown how narrator's self can be positioned via archetypal 'quest stories' (Frank, 1995) which afford sufferers the position of a hero. Changing and manipulating moral positions allows forming new kinds of relationships with audiences - a strategy used in activist projects campaigning on behalf of marginalised groups (Jones, 2015).

Thirdly, the multimodal properties of the medium need to be taken into account in the analysis of online narratives (Page, 2017) since social media platforms allow for combining written texts with audio and video material. In this paper we pay particular attention to how the combination of verbal and visual resources allows the campaign participants to construct different positions for themselves and their audiences as they attempt to 'strike a balance between including enough information to make the utterance interpretable to as many people as possible, while avoiding the inclusion of easily inferred background information' (Scott, 2015: 11). The restricted character space means that in addition to making the online content searchable and scalable, the metadata and images can also be expected to provide contextual information that plays a part in 
positioning tellers and listeners. Sociolinguistic research outside the health communication domain has already shown how the use of hyperlinks, for example, allows Twitter users to position themselves within the context of global knowledge and demonstrate interests and expertise (Dayter, 2016; Page, 2012). Hashtags have also been studied as devices that perform an interpersonal function of negotiating relationships and expressing affiliation with different communities (Zappavigna, 2014; Zappavigna \& Martin, 2017) in addition to functioning as a topic marker (see also 'conversational' vs 'organisational' tagging in Huang et al, 2010). Similarly, photos, and selfies in particular, have been studied as the visual means through which users can continually explore the relationships between one's own personal sense of identity and agency and public self-image (Cover, 2013), as well as broader social representations. In relation to health and illness discourse, Tembeck (2016) shows how selfies constitute an important part of visual self-representational practices that offer a first-person perspective on experiences of illness in order to counter the illness' lack of visibility outside of a medical context.

\section{Methodology}

We initially collected tweets shared with the hashtag \#depression during Depression Awareness Week 2016 (18-22 April). Tweets were collected via DiscoverText using the Twitter Search API described as the best option for historical searching with a hashtag and/or keyword (Milsom, 2015). We focused on UK tweets basing decisions about tweets' geography on the users' selfreported location (to determine the user's location, we relied on the manually specified location in the user's biography and/or the exact location coordinates of the tweet). Although this approach entails weaknesses, as only around 3\% of all tweets are known to contain geographic metadata (Leetaru et al, 2013), limiting our analysis to UK tweets was important for gauging the UK response to Depression Awareness Week. Indeed, this approach means that we have omitted tweets from users who did not explicitly identify a geographic position.

After analysing the frequency of use of all hashtags in the sample, \#WhatYouDontSee emerged as one of the most frequently used hashtags after generic hashtags such as \#depression (our search term), \#mentalhealth and \#DAW2016 (abbreviated from Depression Awareness Week 
2016). Here we report on the analysis of the 438 tweets posted under the \#WhatYouDontSee hashtag. Of all tweets which contained this hashtag, 29 included not just text and metadata but also still images (29), of which 26 were selfies. We excluded videos, as analysis of moving images is beyond the scope of this paper and also, they were too few (2 tweets). Drawing on the research on the social and discourse functions of hashtags in social media discourse (Scott, 2015, 2018; Dayter, 2016) we first examined whether the hashtag was used as part of the sentence or outside the overall syntactic structure of the tweet. We then investigated the two groups in more depth by analysing the combination of the hashtag with lexical and/or visual resources in the creation of specific positions for authors and audiences in the local contexts. Unintegrated hashtags are of particular interest as they can perform a pragmatic function of filling in contextual information by adding 'a layer of activation to certain contextual assumptions [...] guiding the reader's inferential processes' (Scott, 2015: 8). Dayter (2016), for example, found that unintegrated hashtags may 'deliver a crucial piece of information' that indicates identity positioning in relation to a hero persona (of a ballet enthusiast). Having examined the overall use of semiotic resources in each tweet, we grouped results according to audience identity positions emerging from these multi-semiotic combinations.

Ethics approval for this study was obtained from [name after review] University Research Ethics Committee. Although Twitter is a public platform we sought permission to reproduce the tweets that contain selfies from individual Twitter users. We also removed potentially identifying details such as @handles.

\section{Results}

\section{1) \#WhatYouDontSee tweets as testimonies: Positioning audiences as a jury}

The stigma associated with depression is closely linked to the fact that the aetiology of this condition is still poorly understood, with different theories proposed from biology, psychology, and anthropology (Kotliar, 2016). Depression is difficult to understand for others, as there is no obvious 'external cause' unlike in the case of a post-traumatic stress disorder (Wolpert, 2001). Such lack of understanding, according to Wolpert (2001) leads to the fact that depression can be 
seen as 'a sign of weakness', and the individuals who have it as unreliable at work, for example. To counter such perceptions and improve public understanding, the Blurt charity campaign aimed to reveal the 'unseen' everyday realities of people with depression. The hashtag \#WhatYouDontSee proposed by the charity allows potential campaign participants to reveal hitherto unknown issues, either as a statement with generic impersonal plural ('what one doesn't see') or by addressing a putative other or others (singular or plural 'you') and thereby position themselves as witnesses of reported events and circumstances.

In response to the Blurt charity's exhortation to 'Blurt it out', campaign participants used the hashtag to introduce statements describing negative experiences and accounts of emotional and physical pain. In the examples below, the hashtag is integrated into the sentence - it serves as the topic whereas the tweet can be interpreted as commentary on that topic (Huang et al, 2010). While some of the posts indicate the subjective position of the speaker via first person pronouns (e.g. [1]), the majority of tweets in this category express self-reference indirectly via generic 'you' (as in [2], [4], [5]) that stands for a range of individuals that the speaker identifies with, similarly to generic 'one' (Moltmann, 2010). The position of a witness in such tweets therefore also metonymically represents others who have lived through similar situations (see also 'people' in [1]).

[1] \#WhatYouDontSee are the people suffering for years without knowing what is wrong. 8yrs I didn't know; I thought I was a freak \#depression - 19/04/2016

[2] \#WhatYouDontSee is how worthless you feel, a burden to your family. Depression and \#anxiety make you feel shame and guilt at every turn - 21/04/2016

By detailing the 'unseen' struggles experienced by depression sufferers as a group, the tweets invoke the genre of testimony - a mode of storytelling that functions to bring to light a transgression or suffering for the purposes of eliciting empathy and to get accountable those involved (Jones, 2015; McLagan, 2006). As Frank (1995: xii) argues, patient stories are resistance narratives, in that '[i]n stories, the teller not only recovers her voice; she becomes a witness to the conditions that rob others of their voices. When any person recovers his voice, many people 
begin to speak through that story'. This is particularly visible in the tweets that use a collective 'we'/ 'us' [3] to refer to the group.

[3] \#WhatYouDontSee is how hurt we are when you say 'you've got no reason to be unhappy' \#depression doesn't need a 'proper' reason to plague us - 22/04/2016

Testimonials afford the tellers the positions of witnesses while positioning audiences as judges or jury. Designed to show that the suffering is legitimate by offering evidence for public judgement, testimonials often build 'extreme case formulations' (Pomerantz, 1986), such as 'at every turn' [2], 'for years' [1] into the presentation of evidence in order to objectify the claims made.

As one of the purposes of testimony is to bring justice, the tellers typically refer to the causes of their suffering and/or name 'the perpetrators'. Some tweets specify either depression (the subject of the clause in [2]), the putative addressee (in [3]) or other people (such as 'friends' in [4] below) as the parties responsible for the suffering caused:

[4] \#WhatYouDontSee when you feel that your so called friends don't understand what \#depression is - 19/04/2016

Other posts refer to the causes and actors only indirectly. This is done through references to the social aspects of suffering such as imbalances in relationships with family members or co-workers (e.g. [5]), and the negative social consequences of living with depression such as feelings of exclusion and isolation.

[5] \#WhatYouDontSee is that you can't be honest about your \#depression at work as it might be used against you -19/04/2016

Instead of naming the 'perpetrators' to hold them accountable as is done in public testimonies, tweets such as [5] invite moral judgement by describing the types of social situations which cause the tellers pain. By bearing witness to how people with depression avoid disclosing the condition to one's social network or at a workplace because of stigma, the posts invite potentially any reader to see themselves as a bystander who lets it happen. As a result, such Twitter testimonies construct an audience who is simultaneously 'shamed' for allowing the stigmatisation to happen 
and called to hold one another accountable to prevent further suffering (Jones, 2015; McLagan, 2006).

By positioning the audiences as jury that is presented with evidence of suffering and injustice, posts in this category represent a 'broadcasting' disclosure (Corrigan \& Rao, 2012) with the aim

of educating the public about a condition and the impact of stigma on the everyday lives of individuals. Due to affordances of searchability and scalability Twitter users can address both members of their personal network (singular you in \#WhatYouDontSee) and the public at large (plural second person pronoun) who would see the post through sharing, liking or hashtag trending. At the same time, such microblogs propose a bond (Zappavigna, 2015) to fellow sufferers around the WhatYouDontSee hashtag (as well as through the use of inclusive lexis). Such tweets therefore simultaneously invite further testimonies from individuals with depression and encourage validation of reported experiences by the public at large. In order to establish whether these audience identity positions (as a jury and fellow sufferers) are taken up by other Twitter users, we examine reactions to these tweets (analysis in subsection 3 below).

\section{2) \#WhatYouDontSee tweets as confessions: Positioning audiences as confidantes}

Other tweets in our corpus explicitly focus on detailing personal experiences and reporting specific facts about the author of the post. By disclosing personal circumstances, the posts invoke the genre of confession - a narrative that reveals intimate details of a person's life and is driven by 'a desire to tell another who one is' (Taylor, 2008: 250). In some examples such as [6], the hashtag is followed by a hyperlink to a personal blog where personal experiences and reflections had been recorded ('about me'). Whereas in the above examples, the hashtag was used to structure a shared story, here it used as a reference - a quick introduction to shared story by means of a one-line reference to it (De Fina \& Georgakopoulou, 2012: 110).

[6] For \#Depression Awareness Week, here's an old (but still true) post about me and \#WhatYouDontSee (link to post on personal blog) - 18/04/2016 
Other tweets in this category use the hashtag outside the syntactic structure. In examples [7] and [8], the hashtag contributes contextual information about this particular campaign to what is already implicitly communicated via the linguistic content of the tweet and the hashtag \#depression.

[7] Jumping from feeling like I could take on the world to feeling utterly lethargic and down multiple times a day \#WhatYouDontSee \#depression - 19/04/2016

[8] Not having the energy to eat or get undressed after work as so tired from wearing my 'mask' all day \#WhatYouDontSee \#depression \#DAW2016 - 18/04/2016 In addition to tweets disclosing negative circumstances of living with depression, some posts include statements about 'the look' of depression as a response to the campaign's exhortation 'to challenge the stigma around what depression 'should' look like, and show the world that ANYONE can be affected by poor mental health' (Blurt, 2016). Tweets such as [9] below contain non-disclosive informative statements - assertive speech acts that do not obviously disclose information about the author (Dayter, 2016). However, since the campaign invites potential participants to provide personal accounts we can expect that authors of the above tweets disclose information about themselves, and signal this by using \#WhatYouDontSee in addition to \#depression.

[9] Behind each smile, attitude \& walk is a truck-load of motivation to conquer self-doubt and \#depression \#WhatYouDontSee - 18/04/2016

Other tweets about the 'look' of depression use hashtags as contextual cues for selfies of smiling individuals. In some of these tweets, a selfie is accompanied only by the hashtag placed centrally in the body of the image, without any written statement [10]. Scott (2018: 58) argues that hashtags 'sub-divide into uses which contribute to the derivation of the explicit meaning and those which contribute to what is implicitly communicated'. In this case [10], the hashtag guides the addressee to the intended explicit content (rather than contributing to what is already implicitly communicated). Without the inclusion of the hashtag \#WhatYouDontSee in the image the reader would not be able to understand the intended content (Scott, 2018). 
Figure 1. Example 10.

Follow

\section{\#whatyoudontsee \#depression}

\#depressionawarenessweek \#blurtfoundation

alltheunheardvoices.wordpress.com/2016/04/

$19 /$ wha...

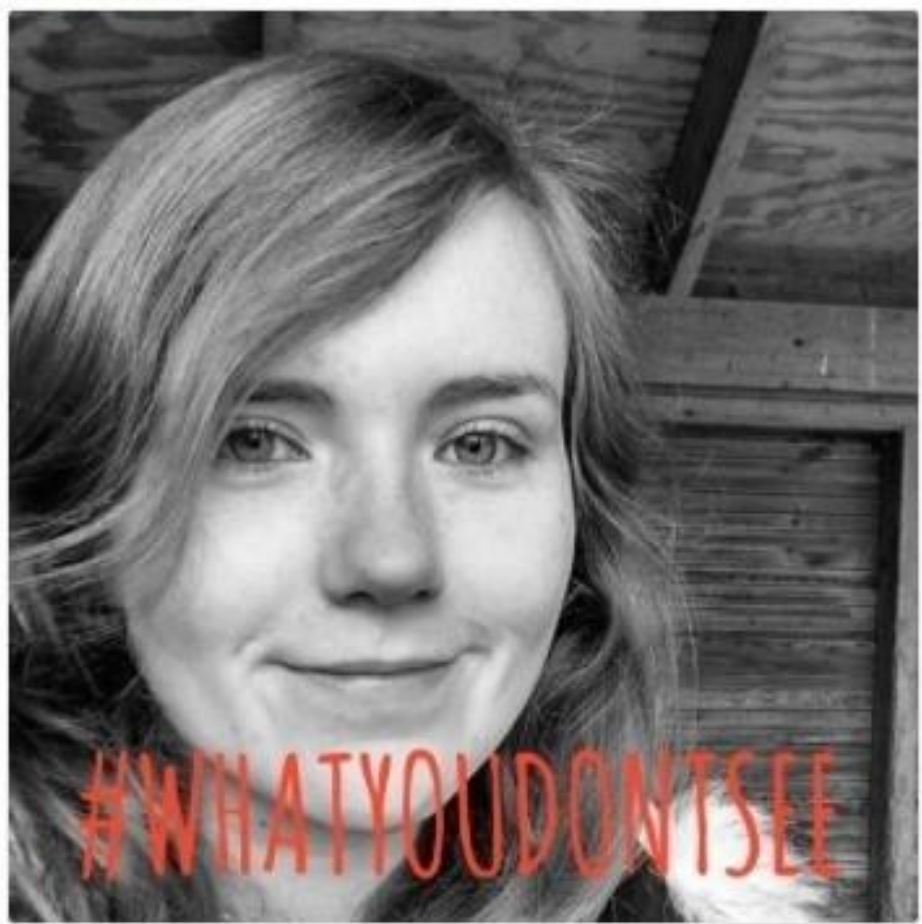

4:13 AM - 19 Apr 2016

2 Retweets 2 Likes 906

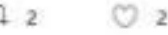

On the one hand, the hashtag in [10] signals identification with the cause and is aimed at construing 'ambient affiliation' (Zappavigna, 2015: 276) with Twitter users who participate in the campaign. Tweets with such selfies therefore resemble protest avatars - a combination of photos, icons, and/or graphics which social media users temporarily adopt as their profile pictures as an act of displaying solidarity with a cause. Such tweets can also be seen as constituting a selfie protest, a practice where individuals emulate public demonstrations by using selfies that depict themselves holding up a self-made protest sign or contain a hashtag encapsulating the 
movement's message or claim (for example, using \#BringBackOurGirls with the profile picture). On the other hand, selfies depicting smiling individuals in our study are more than an expression of solidarity as they are self-portraits by individuals with mental illness, in line with the activist acts of coming-out as being invisibly ill or disabled (Tembeck, 2016). In such cases, selfies are used as visual resources for self-disclosure whereas the second person pronoun in the hashtag is used to address the confidante/s.

In such visual confessions the intimacy of written revelations (such as disclosing negative experiences above) is replaced by the visual representation of the author and the intimacy generated by the power of the direct gaze. In posts that contain selfies accompanied only by the \#WhatYouDontSee hashtag, the deictic centre of the narrator is not established linguistically but emerges from the photo itself. The direct gaze on the same level as the viewer establishes an intimate distance positioning the author of the tweet (the photographer behind the image) as an agent, addressing the reader directly. These are 'demand' images as they invite a relationship/conversation with the viewer (Kress \& van Leeuwen, 2006). Images such as [11] place self-portraits in opposition to statements describing the adverse experiences. The text is embedded into the photo to the right of the self-portrait and therefore can be read as the new information (Kress \& van Leeuwen, 2006) whereas the self-portrait is the given (already known). Such composition presents the selfie author as the sayer of the text granting them agency despite the metaphorical representation of depression as an (unstoppable) force in the text.

Figure 2. Example 11. 


\section{My \#depression \#mhelfie for \\ @theblurtfoundation 's \#DAW2016 campaign. \\ Follow the \#whatyoud... ift.tt/1qB9RNX}

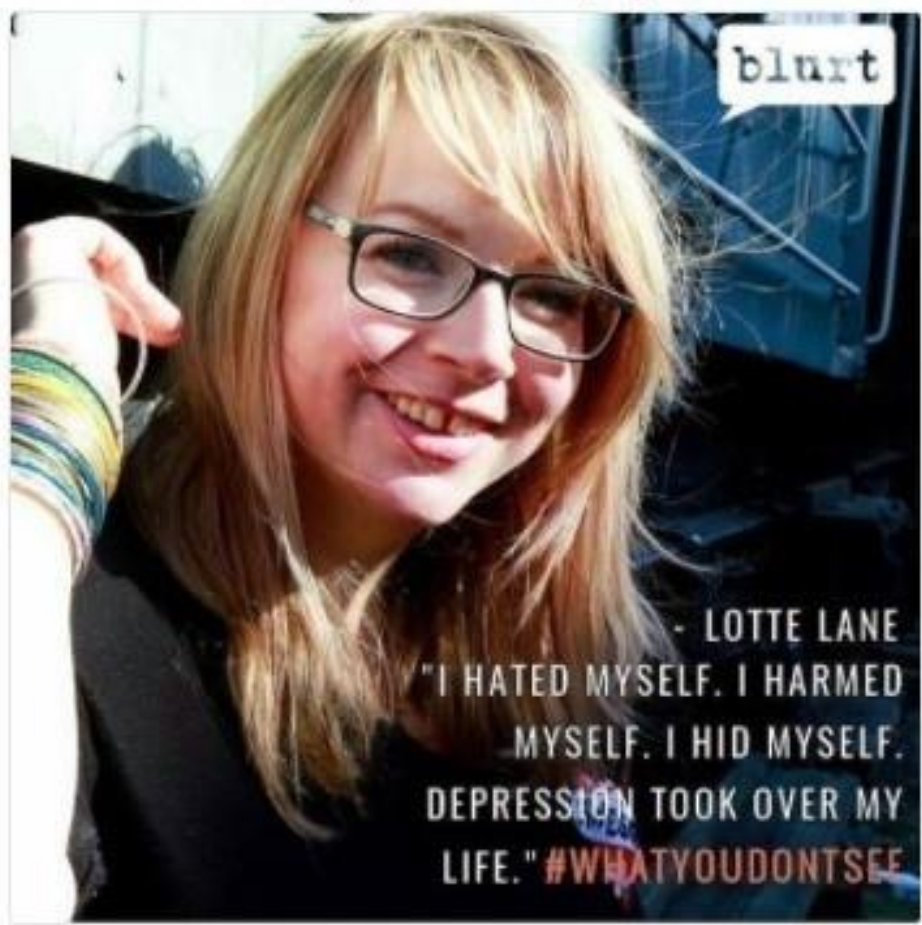

$3: 42$ AM - 18 Apr 2016

3 Retweets 2 Like:

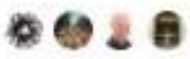

$173 \quad O_{2}$

Reflecting on the practices of using personal photos online, Senft (2008) uses the term 'grab' to refer to the visual arrest generated by a spectacle and connects this affective experience to the physical experience of being touched. Depression Awareness selfies capitalise on such 'grab' properties of selfies as part of the broadcasting disclosure strategy that aims to reach maximum visibility. Such use of self-portraits has been part and parcel of patient advocacy practice, particularly in the case of breast cancer activism (e.g. Dykstra, 1995). While cancer advocacy images deliberately include signs of illness (e.g. photos of individuals with no hair in contrast with the practice of using wigs to hide the side effects of chemotherapy), the self-portraits of smiling individuals for the \#WhatYouDontSee campaign draw attention to the difficulty of disclosing the 
condition to one's social networks and the resulting practice of hiding it behind a cheerful attitude ('the mask').

Self-portraits of smiling individuals also emulate the norms of social media selfies characterized by largely 'phatic' and 'polite' engagement in interaction (Varis \& Blommaert, 2015). Koteyko and Hunt (2015), for example, show how Facebook users with diabetes avoid stories that disclose the negative sides of diabetes management to their network. One campaign participant alluded to such social media practices and the associated difficulties of disclosing a stigmatised condition by providing a collage of his Facebook photos that depict exclusively happy occasions and smiling people/family members (tagged with \#WhatYouDontSee with the tweet content reading 'From Facebook by my wife for me'). Depression Awareness week tweets with such selfies can therefore be seen as part of the practices that politicise 'discourses about how people ought to represent, document and share their behaviour' (Senft \& Baym, 2015: 1589) and invite us to bear witness not only to individual stories of suffering as reflections of socio-political realities but also to the role of mediation (Hall, 2016).

\section{3) Reactions to the campaign: Taking up the audience positions}

The multiple audience positions afforded by the campaigners' tweets can be traced in reactions to the campaign (recorded and retrieved due to the use of the same hashtag). Pursuing the interactional goal of praising the campaign, its organisers, and contributors, such reactions integrate the hashtag syntactically as a modifier in the noun phrase to refer to the online aggregation in the form of the feed. Authors of such tweets take up the positions of confidantes and jury offered by the campaigners' tweets by validating the confessed experiences and assessing the testimonies through their positive evaluation:

[12] Incredibly moved reading \#WhatYouDontSee responses - \#depression affects so many of us yet it's still stigmatised and silenced - 21/04/2016

[13] Delighted to see \#WhatYouDontSee trending today, and all the brilliant contributions. Great work by @BlurtAlerts! \#depression - 18/04/2016 
Such evaluations ( [12] and [13]) reflect the common practice of social media annotation where metadata is used by individuals to make each other aware of trending topics (Page, 2010). Other tweets simultaneously acknowledge witnessing the experiences of others via reading the thread (and the confessions and testimonials therein) and adopt the position of a fellow sufferer by stating that the campaign tweets voiced their own experiences:

[14] I recognise so much in this thread. \#whatyoudontsee \#depression (link to personal profile) - 18/04/2016

In the examples cited below, the aesthetic of exposure enabled via selfies or linguistic selfdisclosure discussed in previous sections takes the form of a reference to the content aggregated via the two hashtags:

[15] I discovered the hashtag \#WhatYouDontSee re \#Depression \& seeing strangers articulate aspects of my interior life' - 18/04/2016

[16] This entire \#WhatYouDontSee thread re \#Depression = me. I've been deep in latest cycle of MH hell for almost 1 month (link to personal status update) - 18/04/2016

References to the hashtag thread and a hyperlink to one's profile detailing recent Twitter activity function both as markers of intertextuality and as resources for self- and otherpositioning, and therefore provide another example of how metadata allows social media users to enact specific identities. Reference to the hashtag feed invites audiences to view the accounts provided by others rather than (only) rely on the author's own textual descriptions, while the link to one's personal status update provides further information to corroborate the author's negative stance ('MH hell'). In such instances, the use of metadata enables new forms of self-disclosure based on the combination of lexical and metadata resources.

\section{Discussion}

The study set out to examine how Twitter users position themselves and their audiences when they represent their private experiences of illness in the context of mental health advocacy. The approach to narrative advocated in interactional sociolinguistics (Bamberg, 2011) invites us to consider stories of personal illness experience not only as therapeutic and meaning-making 
resources (Bury, 2001; Charmaz, 2002) but also as performances that construct specific relationships with the audience (Wortham, 2000) and call for attention to the different semiotic resources used in the act of expression (Georgakopoulou, 2016). While this approach has so far been used to highlight the interactional dynamics and underlying institutional structures in illness narratives elicited via one-to-one interviews (e.g. Miczo, 2003), applying this analytical lens to Twitter data has helped us to reveal how interactional positioning is accomplished in multisemiotic social media narratives about a stigmatised condition. Our analysis has shown that while tweets contain partial rather than full-fledged tellings, such small stories nevertheless invoke two types of canonical narratives. These two narrative genres of testimony and confession and the positions that they make available for tellers and their audiences are evoked not only through the choice of linguistic devices but also through the use of images and metadata. In what follows we discuss in more detail the role of hashtags, hyperlinks and images as cues for interactional positioning in the social media context.

The evolving pragmatic function of unintegrated hashtags has been examined by Scott (2018) who argues that they play an important contextualising function in the social media setting where audiences have to be 'imagined' (Marwick \& boyd, 2010). The analysis of tweets in our corpus has shown how \#WhatYouDontSee was used to add information crucial for understanding the content of the tweet (particularly visible in examples where the hashtag is superimposed on a photograph without any further information) and to provide a piece of information that clarifies the utterance. Such uses, we argue, point to the role of the hashtag as a contextualisation device that indexes the moral stance (Och \& Capps, 2001) and in this way allows recipients to differentiate tweeting about negative experiences for a mental health advocacy campaign from a common practice of social media 'venting'. In structural and semantic terms, \#WhatYouDontSee tweets constitute third party complaints - expressions of grievance to an audience not immediately responsible for committing the complainable (Dayter, 2016). While such 'trouble talk' (Goffman, 1981) typically invites response in the form of expression of support (rather than acceptance or remediation as in the case of a direct complaint), the written and visual testimonies and confessions tagged with \#WhatYouDontSee are designed to 'grab' our attention and direct it to the ethical perception of the narrator. Such 
online revelations do not seek support from an interlocutor and indeed were met with praise rather than commiserations, as can be seen from the reactions to the campaign. Through the combination of the hashtag with the linguistic representation of pain and suffering, or the visual spectacle of selfie such tweets address an imagined witness who will align with the stance of the author and 'validate both a confessed experience, and the larger social issues to which such an experience points' (Hall, 2016: 240). The hashtag \#WhatYouDontSee therefore provides important contextual assumptions that ensure that a telling will be heard as an act of raising awareness, even if this is not explicitly flagged in the rest of the tweet.

Our analysis has shown how campaign participants mobilize the hashtag alongside linguistic and visual resources to construct different relationships with their networked audiences. By integrating the hashtag semantically and syntactically to introduce written accounts that detail everyday struggles of depression sufferers as a group of Twitter users adopt the position of an eyewitness who is presenting evidence in front of a jury (the audience). In such Twitter testimonials, the hashtag is used not only to structure statements (signalling the topic of the tweet), but also to enact communal bonds (Zappavigna, 2015) with others who would use the hashtag in the same way (in this case, fellow sufferers).

Using the hashtag as a reference (to a shared story) to contextualise both written statements about first person experiences and selfies allowed the participants to position the audiences as confidantes. Some of these mediatised confessions rely on hyperlinks to personal profile pages on Twitter or external personal websites. Hyperlinks to extended narratives allow recipients a greater opportunity to understand the author's preferences and symbolic connections, and in this way complement the intimacy of the written and visual self-disclosures. Self-disclosive posts in this category also use selfies as small stories that capture the moment in the life of the teller (Georgakopoulou, 2016) and help create narratives that reveal something 'deeply personal and not always obvious about the confessant' (Hall, 2016: 229). The agentic stance in the selfie can be directly contrasted with the position of 'self as an undergoer' (Bamberg, 2011: 106) and provides further cues that the telling is not a complaint story. At the same time, selfies do not only serve the representational function (representing the authors as objects) but also 
allow the campaign participants to articulate their perspective and negotiate it in public - they are also technologies for seeing as (Zhao \& Zappavigna,2015). Selfies tagged with \#WhatYouDontSee are therefore closely related to another social media practice dubbed 'cue card confessions' - autobiographical videos disclosing suffering with a political dimension such as sexism, racism or bullying where individuals use cue cards to tell a story (Hall, 2016).

The positions of jury, confidantes, and fellow sufferers constructed in the participants' tweets were taken up in tweets that reacted to the campaign. The uptake of the campaign messages triggered a new wave of circulation, and a new type of self-disclosures that identified with previously voiced experiences via references to the hashtag feed. Such acts of 'networked reflective solidarity' (Senft, 2008) can also be traced through the use of selfies, as selfies tagged with \#WhatYouDontSee during the Depression Awareness Week led to a specific practice of posting 'mhelfies' (mental health selfies) by people affected by a mental health issue. As a result, the process of producing the campaign tweets and reacting to the content of such tweets became a form of "witnessing" (Hall, 2016) that conveys both the authority to stand as a witness as well as the rights to grant the validity to someone else's experience.

The point emphasised throughout our analysis is that metadata, written statements, and/or images form multisemiotic texts characterised by extensive relation between the elements: such compositions would be incomplete if one of the elements were to be omitted (Kress \& van Leeuwen, 2006). Further analytic attention to the uses of visuals and metadata alongside linguistics resources for signalling interactional positioning in different social media contexts (e.g. dyadic communications not examined here due to space limitations, or conversations in groups such as Facebook groups) is necessary to illuminate how platform affordances influence the pragmatics of digital illness narratives. Such projects would be well placed to complement and advance the current focus on the representational function of narratives (i.e. content analyses dominating the field of in e-health) by uncovering how networked stories of illness allow individuals 'to relate, to tell, to reveal, to reach out on a human level' (McKechnie, 2014: 122). 


\section{References}

Adami, E. \& Jewitt, C. (2016) Special Issue: Social media and visual communication. Visual Communication 15 (3): 263-270.

Bamberg, M. \& Georgakopoulou, A. (2008) Small stories as a new perspective in narrative and identity. Text and Talk 28: 377-396.

Bamberg, M. (2011) Narrative practice and identity navigation. In J. A. Holstein \& J. F. Gubrium (eds.), Varieties of Narrative Analysis, 99-124. London: Sage Publications.

Bennett, L. W. \& Segerberg, A. (2013) The logic of connective action: Digital media and the personalization of contentious politics. Cambridge: Cambridge University Press.

Benson, A., Secker, J., Balfe, E., Lipsedge, M. Robinson, S. \& Walker, J. (2003) Discourses of blame: Accounting for aggression and violence on an acute mental health inpatient unit. Social Science \& Medicine 57(5): 917-926.

Blurt (2016) Depression Awareness Week: Our \#WhatYouDontSee campaign, Available at: http://blurtitout.org/2016/04/18/whatyoudontsee/ (accessed 25 April 2016).

Bolander, B. \& Locher, M. (2010) Constructing identity on Facebook: Report on a pilot study. SPELL: Swiss Papers in English Language and Literature 24: 165-187.

boyd, d. (2010) 'Social network sites as networked publics: Affordances, dynamics, and implications' In Papacharissi, Z. (ed) Networked self: Identity, community, and culture on social network sites (pp. 39-58). New York and London: Routledge.

boyd, d. \& Ellison, N. (2007) Social network sites: Definition, history, and scholarship. Journal of Computer-Mediated Communication 13 (1): 210-230.

boyd, d. \& Heer, J. (2006) Profiles as conversation: Networked identity performance on Friendster. In: HICSS-39: Proceedings of the Hawaii International Conference on System Sciences, Kauai, pp. 1-10.Bucholtz, M. \& Hall, K. (2005) Identity and interaction: A sociocultural linguistic approach. Discourse Studies 7(4-5): 585-614.

Bury, M. (2001) Illness narratives: Fact or fiction? Sociology of Health \& IIIness 23(3): 263-285.

Charmaz, K. (2002) Stories and silences: Disclosures and self in chronic illness. Qualitative Inquiry 8(3): 302-328. 
Corrigan, P. W. \& Rao, D. (2012) On the self-stigma of mental illness: Stages, disclosure, and strategies for change. The Canadian Journal of Psychiatry 57(8): 464-469.

Cover, R. (2013) 'Becoming and belonging: Performativity, subjectivity, and the cultural purposes of social networking' In Poletti A. \& Rak, J. (eds) Identity technologies: Constructing the self online (pp. 55-69). Madison: The University of Wisconsin Press.

Crowe, M. (2000) Constructing normality: A discourse analysis of the DSM-IV. Journal of Psychiatric and Mental Health Nursing 7(1): 69-77.

Dayter, D. (2016) Discursive self in microblogging: Speech acts, stories and self-praise. Amsterdam/Philadelphia: John Benjamins.

Dykstra, J. (1995) Putting herself in the picture: Autobiographical images of illness and the body, Afterimage 23: 16-20.

Frank, A. W. (1995) The wounded storyteller: Body, illness, and ethics. Chicago: University of Chicago Press.

Galasinski, D. (2008) Men's discourses of depression. Houndmills, Hampshire and New York: Palgrave Macmillan.

Georgakopoulou, A. (2007) Small stories, interaction and identities. Amsterdam: John Benjamins.

Georgakopoulou, A. (2014) Small stories, transposition and social media: A micro-perspective on the Greek crisis. Discourse and Society 25 (4): 519-539.

Georgakopoulou, A. (2015) Small stories research: Methods-Analysis-Outreach. In De Fina, A. \& Georgakopoulou, A. (eds) The handbook of narrative analysis (pp. 255-272). Malden, MA: Wiley-Blackwell.

Georgakopoulou, A. (2016) From narrating the self to posting self(ies): A small stories approach to selfies. Open Linguistics 2: 300-317.

Goffman, E. (1981) Forms of talk. Philadelphia: University of Pennsylvania Press.

Hall, K. (2016) Selfies and self-writing cue card confessions as social media technologies of the self. Television \& New Media 17(3): 228-242. 
Harré, R. (2012) Positioning theory: Moral dimensions of social-cultural psychology. In Valsiner, J. (ed) The Oxford Handbook of Culture and Psychology (pp. 191-206) New York: Oxford University.

Herring, S. C. (2007) A faceted classification scheme for computer-mediated discourse. Language@Internet. Available at: http://www.languageatinternet.de/articles/761 (accessed 11 April 2018).Horwitz, A. V. \&Wakefield, J. C. (2007) The loss of sadness: How psychiatry transformed normal sorrow into depressive disorder. New York: Oxford University Press.

Huang, J., Thornton, K. \& Efthimiadis, E. N. (2010) Conversational tagging in twitter. In: HT'10: Proceedings of the $21^{\text {st }}$ ACM conference on Hypertext and hypermedia, Toronto, pp. 173-178.

Hughes, D. J., Rowe, M, Batey, M. \& Lee, A. (2012) A tale of two sites: Twitter vs. facebook and the personality predictors of social media usage. Computers in Human Behavior 28(2): 561-569.

Jamison-Powell, S., Linehan, C. Daley, L., Garbett, A. \& Lawson, S. (2012) 'I can't get no sleep’: Discussing \#insomnia on Twitter. In: $\mathrm{CHI} 12$ : Proceedings of the SIGCHI Conference on Human Factors in Computing Systems, New York, pp. 1501- 1510.

Jones, R. H. (2015) Generic intertextuality in online social activism: The case of the It Gets Better project. Language in Society 44(3): 317-339.

Koteyko, N. \& Hunt, D. (2016) Performing health identities on social media: An online observation of Facebook profiles. Discourse, Context \& Media, doi: 10.1016/j.dcm.2015.11.003.

Kotliar, D. M. (2016) Depression narratives in blogs: A collaborative quest for coherence. Qualitative Health Research 26(9): 1203-1215.

Kress, G. \& van Leeuwen, T. (2006) Reading images: The grammar of visual design. London: Routledge.

Kvaale, E. P., Gottdiener, W. H. \& Haslam, N. (2013) Biogenetic explanations and stigma: A meta-analytic review of associations among laypeople. Social Science \& Medicine 96: 95103. 
Labov, W. (1972) Language in the inner city. Philadelphia: University of Pennsylvania Press. Leetaru, K. H., Wang, S., Cao, G., Padmanabhan, A. \& Shook, E. (2013) Mapping the global Twitter heartbeat: The geography of Twitter. First Monday 18(5-6), http://dx.doi.org/10.5210/fm.v18i5.4366.

Mancini, M. A. \& Rogers, R. (2007) Narratives of recovery from serious psychiatric disabilities: A Critical Discourse Analysis. Critical Approaches to Discourse Analysis across Disciplines 1(2): 35-50.

Marwick, A. E. \& boyd, d. (2010) I tweet honestly, I tweet passionately: Twitter users, context collapse, and the imagined audience. New Media and Society 13(1): 114-133.

McKechnie, C. (2014) Anxieties of communication: the limits of narrative in the medical humanities. Medical Humanities 40: 119-124.

McLagan, M. (2006). Introduction: Making human rights claims public. American Anthropologist 108: 191-195.

Meikle, G. (2018) The Routledge Companion to Media and Activism. London: Routledge.

Milsom, B. (2015) Twitter API vs Firehose, Available at: https://www.echosec.net/twitter-apivs-firehose/ (accessed 19 April 2016).

Miczo, N. (2003) Beyond the fetishism of words: considerations on the use of the interview to gather chronic illness narratives. Qualitative Health Research 13(4): 469-90.

Moltmann, F. (2010) Generalizing detached self-reference and the semantics of generic one. Mind and Language 25: 440-473.

Murthy, D. (2012) Towards a sociological understanding of social media: Theorizing Twitter. Sociology 46(6): 1059-1073.

National Institute for Health and Care Excellence (2015) Depression, Available at: http://cks.nice.org.uk/depression\#!backgroundsub:1 (accessed 1 June 2016).

Neuhauser, L. \& Kreps, G. L. (2003) Rethinking communication in the e-health era. Journal of Health Psychology 8(1): 7-23.

Ochs, E. \& Capps, L. (2001) Living narrative: Creating lives in everyday storytelling. Cambridge, MA: Harvard University Press. 
Page, R. (2010) Re-examining narrativity: Small stories in status updates. Text \& Talk 30(4): 423444. doi:10.1515/text.2010.021.

Page, R. (2012) Stories and social media: Identities and interaction. London, England: Routledge.

Page, R. (2017). Narratives Online: Shared Stories and Social Media Controversies, Cambridge: CUP.

Page, R., Harper, R. \& Frobenius, M. (2013) From small stories to networked narrative: The evolution of personal narratives in Facebook status updates. Narrative Inquiry 23(1): 192-213.

Park, M., Cha, C. \& Cha, M. (2012) Depressive moods of users portrayed in twitter. Presented at: ACM SIGKDD Workshop on Healthcare Informatics, Beijing, China.

Pomerantz, A. (1986) Extreme case formulations: A way of legitimizing claims. Human Studies 9: 219-229.

Read, J. \& Law, A. (1999) The relationship of causal beliefs and contact with users of mental health services to attitudes to the 'mentally ill'. International Journal of Social Psychiatry 45: $216-229$.

Reali, F., Soriano, T. \& Rodríguez, D. (2016) How we think about depression: The role of linguistic framing. Revista Latinoamericana de Psicología 48(2): 127-136.

Scott, K. (2015) The pragmatics of hashtags: Inference and conversational style on Twitter. Journal of Pragmatics 81: 8-20.

Scott, K. (2018). "Hashtags work everywhere": The pragmatic functions of spoken hashtags. Discourse, Context, and Media 22: 57-64.

Seargeant, P. \& Tagg, C. (2014) The Language of social media: Identity and community on the Internet. Basingstoke: Palgrave Macmillan.

Senft, T. (2008) Camgirls: Celebrity and community in the age of social networks. New York, NY: Peter Lang.

Senft, T. M. \& Baym, N. K. (2015) What does the selfie say? Investigating a global phenomenon. International Journal of Communication 9:: 1588-1606. 
Sokratis D., Stevens, S., Serfaty, M., Weich, S. \& King, M. (2004) Stigma: the feelings and experiences of 46 people with mental illness. The British Journal of Psychiatry 184 (2) 176-181.

Taylor, C. (2008) The culture of confession from Augustine to Foucault. London: Routledge.

Tembeck, T. (2016) Selfies of ill health: Online autopathographic photography and the dramaturgy of the everyday. Social Media + Society, 1-11, doi: $10.1177 / 2056305116641343$.

Thomas, W. I. \& Znaniecki, F (1958) [1918_/1920] The Polish Peasant in Europe and America Volumes I and II. New York: Dover Publications.

Tucker, I. (2009) 'This is for life': A discursive analysis of the dilemmas of constructing diagnostic identities. Forum Qualitative Sozialforschung / Forum: Qualitative Social Research 10(3), doi:10.17169/fqs-10.3.1376.

Varis, P. \& Blommaert, J. (2015) Conviviality and collectives on social media: Virality, memes, and new social structures. Multilingual Margins 2(1): 31-45.

Whittier, N. (2012) 'The politics of coming out' In Goodwin, J., Kutz-Flamenbaum, R., Maney, G. \& Rohlinger, D. (eds) Strategies for Social Change (pp. 145-169) Minneapolis: University of Minnesota Press.

Wolpert, L. (2001) Stigma of depression - a personal view. British Medical Bulletin 57(1): 221224.

World Health Organization (2017) Depression Fact sheet. Available at: http://www.who.int/mediacentre/factsheets/fs369/en/ (accessed 5 August 2017).

Wortham, S. (2000) Interactional positioning and narrative self-construction. Available at: https://repository.upenn.edu/gse pubs/92 (accessed 11 April 2018).

Zappavigna, M. (2014) 'CoffeeTweets: Bonding around the bean on Twitter' In Seargeant, P. \& Tagg, C. (eds) The Language of Social Media: Identity and Community on the Internet (pp. 139-160). London: Palgrave Macmillan.

Zappavigna, M. (2015) Searchable talk: the linguistic functions of hashtags. Social Semiotics 25(3): 274-291. 
Zappavigna, M. \& Martin, J. R. (2017) \#Communing affiliation: Social tagging as a resource for aligning around values in social media. Discourse, Context and Media, http://dx.doi.org/10.1016/j.dcm.2017.08.001

Zhao, S. \& Zappavigna, M. (2017) Beyond the self: Intersubjectivity and the social semiotic interpretation of the selfie. New Media \& Society, https://doi.org/10.1177/1461444817706074 